\title{
Erratum: Closed expression for the pair vibrational correlation energy of a uniform distribution of single-nucleon levels [Phys. Rev. C 94, 054328 (2016)]
}

\author{
K. Neergård
}

Q (Received 21 September 2018; published 19 November 2018)

DOI: 10.1103/PhysRevC.98.059901

The caption of Fig. 2 contains an incorrect statement. As already indicated correctly in the text (see page 5, last paragraph in the second column), the caption should instead state that the plotted function is proportional to " ... and valence space dimension $4 \Omega$.” (rather than proportional to “ ... and pair coupling constant $G$. .).

Accordingly, the second sentence in the caption of Fig. 2 should read, "This function is proportional to the two-neutron or two-proton or, for $N=Z$, the neutron-proton pair vibrational correlation energy for a constant single-nucleon level spacing $1 / g$ and valence space dimension $4 \Omega . "$ 